\title{
The GeV Gamma-Ray Emission Detected by Fermi-LAT Adjacent to SNR Kesteven 41
}

\author{
Bing Liu ${ }^{1}$, Yang Chen ${ }^{1,2}$, Xiao Zhang ${ }^{1}$, Gao-Yuan Zhang ${ }^{1}$, Yi Xing ${ }^{3}$ \\ and Thomas G. Pannuti ${ }^{4}$ \\ ${ }^{1}$ Department of Astronomy, Nanjing University, \\ 163 Xianlin Avenue, Nanjing 210023, China \\ ${ }^{2}$ Key Laboratory of Modern Astronomy and Astrophysics, \\ Nanjing University, Ministry of Education, \\ Nanjing 210093, China \\ ${ }^{3}$ Key Laboratory for Research in Galaxies and Cosmology, \\ Shanghai Astronomical Observatory, Chinese Academy of Sciences, \\ 80 Nandan Road, Shanghai 200030, China \\ ${ }^{4}$ Space Science Center, Department of Earth and Space Sciences, \\ Morehead State University, \\ 235 Martindale Drive, Morehead, KY 40351, USA
}

\begin{abstract}
Gamma-ray observations for Supernova remnant (SNR)-molecular cloud (MC) association systems play an important role in the research on the acceleration and propagation of cosmic-ray protons. Through the analysis of 5.6 years of Fermi-Large Area Telescope observation data, here we report on the detection of a gamma-ray emission source near the SNR Kesteven 41 with a significance of $24 \sigma$ in $0.2-300 \mathrm{GeV}$. The best-fit location of the gamma-ray source is consistent with the MC with which the SNR interacts. Several hypotheses including both leptonic and hadronic scenarios are considered to investigate the origin of these gamma-rays. The gamma-ray emission can be naturally explained by the decay of neutral pions produced via the collision between high energy protons accelerated by the shock of Kesteven 41 and the adjacent MC. The electron energy budget would be too high for the SNR if the gamma-rays were produced via inverse Compton (IC) scattering off the Cosmic Microwave Background (CMB) photons.
\end{abstract}

Keywords. acceleration of particles, gamma rays: observations, supernova remnants ,ISM: individual (Kes 41)

\section{Introduction}

Cosmic rays (CRs) are highly energetic particles comprised primarily of protons and atomic nuclei with a small fraction $(\lesssim 1 \%)$ of leptons. Since CRs were discovered by Victor Hess in 1912, the origin of CRs remains one of the most debated issues in high energy astrophysics. As results of stellar explosions, supernova remnants (SNRs) are widely believed to be the main accelerators of Galactic CRs (e.g., Ginzburg \& Syrovatskii 1969). In recent years, a lot of breakthroughs have been made in the study of the SNRs and the origin of Galactic CRs through gamma-ray observations. For instance, gamma-ray emissions produced through $\pi^{0}$-decays have been detected from several SNRs close to MCs (e.g., Ackermann et al. 2013). There are generally two scenarios on the hadronic gamma-rays associated with these SNR-MC systems. In one scenario, the $\pi^{0}$-decay emission is suggested to arise from shock-crushed dense clouds where the accelerated protons frozen in the clouds efficiently collide with target cloud gas (e.g., Blandford \& Cowie 1982; 
Uchiyama et al. 2010; Tang \& Chevalier 2014). In the other scenario, the gamma-rays are ascribed to the hadronic interaction between the relativistic protons escaping from the SNR shock and the adjacent MCs (e.g.,Aharonian \& Atoyan 1996; Gabici et al. 2009; Li \& Chen 2010; Ohira et al. 2011). In both scenarios, SNRs interacting with MCs play an important role in the research on acceleration of protons. The hadronic gamma-ray emission from the SNR-MC systems is usually bright in the GeV domain. Several GeVbright SNRs interacting with MCs have recently been discovered with the Large Area Telescope (LAT) on board the Fermi Gamma-ray Space Telescope, such as W51C (Abdo et al. 2009), W28 (Abdo et al. 2010a), W44 (Abdo et al. 2010b), IC 443 (Abdo et al. 2010c), W41 (Castro et al. 2013), RCW 103 (Xing et al. 2014). Additional GeV observations continue to enlarge the sample of hadronic interaction between SNRs and MCs, and here we present a GeV study of another SNR, namely, Kesteven 41 (G337.8-0.1).

Kesteven 41 (here after Kes 41) is a southern-sky SNR interacting with MC. It is centrally brightened in X-rays within a distorted radio shell, therefore, is classified as a thermal composite (or mixed-morphology) SNR. Kes 41 has also been found to be interacting with an adjacent $\mathrm{MC}$, as indicated by the $1720 \mathrm{MHz}$ hydroxyl radical $(\mathrm{OH})$ maser emission detected in the northern radio shell (Koralesky et al. 1998, Caswell 2004). Recently, we found that Kes 41 is associated with a giant MC at a systemic local standard of rest (LSR) velocity of $-50 \mathrm{~km} \mathrm{~s}^{-1}$ and is confined in a cavity delineated by a northern molecular shell, a western concave MC, and a southeastern HI cloud (Zhang et al. 2015). It is logical and meaningful to search for $\mathrm{GeV}$ emission due to the interaction of highenergy particles.

\section{Fermi-LAT Data Analysis and Results}

Using 5.6 years Pass 7 data from Fermi-LAT (Atwood et al. 2009), we select the LAT events in the energy range of $0.2-300 \mathrm{GeV}$, and perform binned likelihood analyses inside a $14^{\circ} \times 14^{\circ}$ region of interest (ROI) centered at the position of Kes 41 .

Source detection. A gamma-ray source (hereafter source A) is detected with a significance of $24 \sigma$ in the $0.2-300 \mathrm{GeV}$ energy range. Its best-fit position is found to be R.A. $(\mathrm{J} 2000)=16^{h} 38^{m} 36^{s} .00$, decl. $(\mathrm{J} 2000)=-46^{\circ} 55^{\prime} 06^{\prime \prime} .96$.

Spectral analysis. Its spectra ranging from 0.2 to $300 \mathrm{GeV}$ can be fitted with a simple power-law model with a photon index of $\Gamma=2.38 \pm 0.03$. The flux is $(9.2 \pm 1.0) \times$ $10^{-11} \mathrm{erg} \mathrm{cm}^{-2} \mathrm{~s}^{-1}$, and the corresponding luminosity is $\sim 1.6 \times 10^{36} d_{12}^{2} \mathrm{erg} \mathrm{s}^{-1}$, where $d_{12}=d / 12 \mathrm{kpc}$ is the distance to the MC associated with SNR Kes 41. See also section 3.1 for an estimate of the flux and luminosity with an exponential cutoff.

Timing analysis. We also apply long-term variability test on source A, using the method introduced in section 3.6 of Nolan et al. $(2012)$. Its Variability Index $\left(T S_{v a r}\right)$, equal to 65.2 , doesn't reach the threshold (98.0 for 69 time bins) to claim a long-term variability. And no periodic signal is detected through our Fermi-LAT aperture photometry analyses.

\section{The Origin of the Detected Gamma-Ray Emission}

\subsection{Emission from a pulsar?}

We fit the spectrum of source A with an exponential cutoff power law model,

$$
d N_{\mathrm{ph}} / d E_{\mathrm{ph}}=K E_{\mathrm{ph}}^{-\Gamma} \exp \left(-E_{\mathrm{ph}} / E_{\mathrm{ph}, \mathrm{cut}}\right)
$$

and obtain the cutoff energy, $E_{\mathrm{ph} \text {,cut }}=4.0 \pm 0.9 \mathrm{GeV}$ and the spectral index, $\Gamma=1.9 \pm 0.1$, similar to those of the detected gamma-ray pulsars (Abdo et al. 2013). The corresponding 


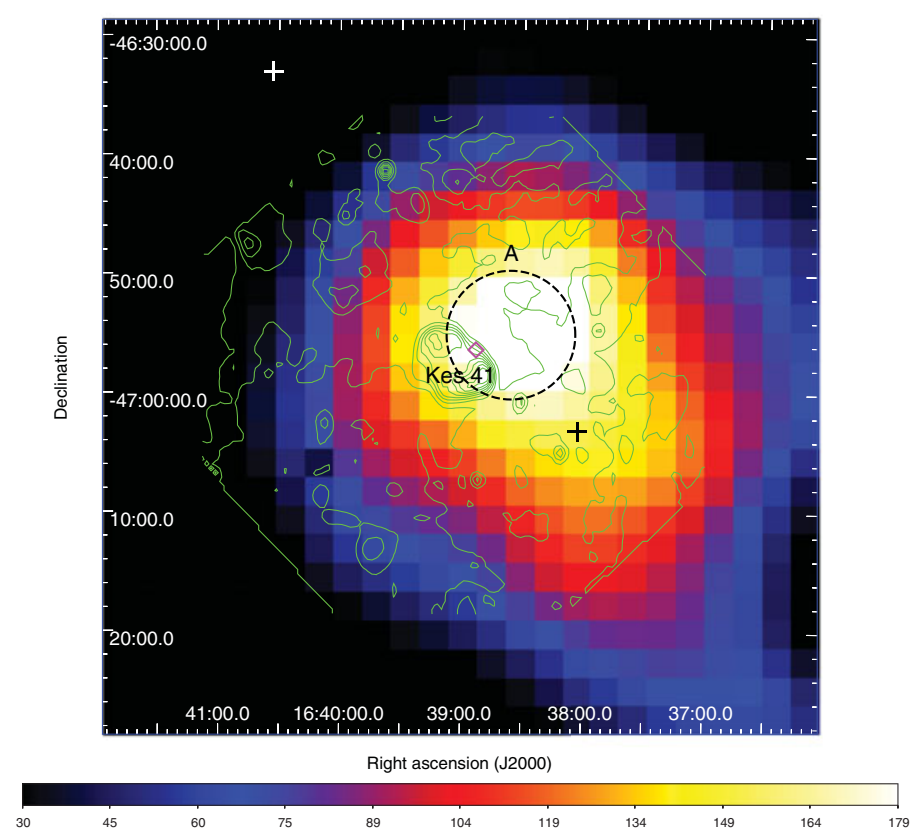

Figure 1. TS map $(2-300 \mathrm{GeV})$ of $1^{\circ} \times 1^{\circ}$ region centered on Kes 41. All sources have been subtracted, except 2FGL J1638.0-4703c (see Liu et al. (2015) for more details). The white cross presents the position of a 2FGL source, the black cross shows the position of 2FGL J1638.0-4703c, and the dashed black circle indicates the $3 \sigma$ error range of the bestfit position for the residual emission found in the Kes 41 region. The image is overlaid with the MOST $843 \mathrm{MHz}$ radio contours (from Whiteoak \& Green (1996)). The magenta diamond indicates the $\mathrm{OH}(1720 \mathrm{MHz})$ maser spot (Koralesky et al. 1998).

flux and luminosity in the energy range $0.2-300 \mathrm{GeV}$ is $(7.5 \pm 0.9) \times 10^{-11} \mathrm{erg} \mathrm{cm}^{-2} \mathrm{~s}^{-1}$, and $(1.3 \pm 0.2) \times 10^{36} d_{12}^{2} \mathrm{erg} \mathrm{s}^{-1}$ respectively. But no known pulsar is within the $3 \sigma$ error circle of the best-fit position of source A. Nonetheless, we can not rule out the possibility that the emission comes from a pulsar.

\subsection{Emission from Particles Accelerated by Kes 41?}

We search the SIMBAD Astronomical Database (Wenger et al. 2000) within the $3 \sigma$ error circle of the best-fit source location, looking for the possible counterpart of the gammaray emission. In addition to Kes 41, only several stars, dark clouds, a young stellar object candidate and an infrared source are known in this region. As shown in Fig.2, the location of source A is consistent with the shock-MC interaction region. Therefore, relativistic particles accelerated by the SNR shock waves are very likely to be responsible for the gamma-ray emission.

Case A. First, we consider the leptonic scenario (case A): gamma-rays are produced by the IC emission of relativistic electrons accelerated by the SNR. We assumed a power-law electron spectrum with an exponential cutoff,

$$
d N_{\mathrm{e}} / d E_{\mathrm{e}} \propto E_{\mathrm{e}}^{-\alpha_{e}} \exp \left(-E_{\mathrm{e}} / E_{\mathrm{e}, \mathrm{cut}}\right) .
$$

In this case, only the cosmic microwave background is considered as the seed photons during the fitting process.

Case B. Next, we consider the hadronic scenario in which these gamma-rays are produced by the $\pi^{0}$-decay after the inelastic collision of the shock accelerated protons with 


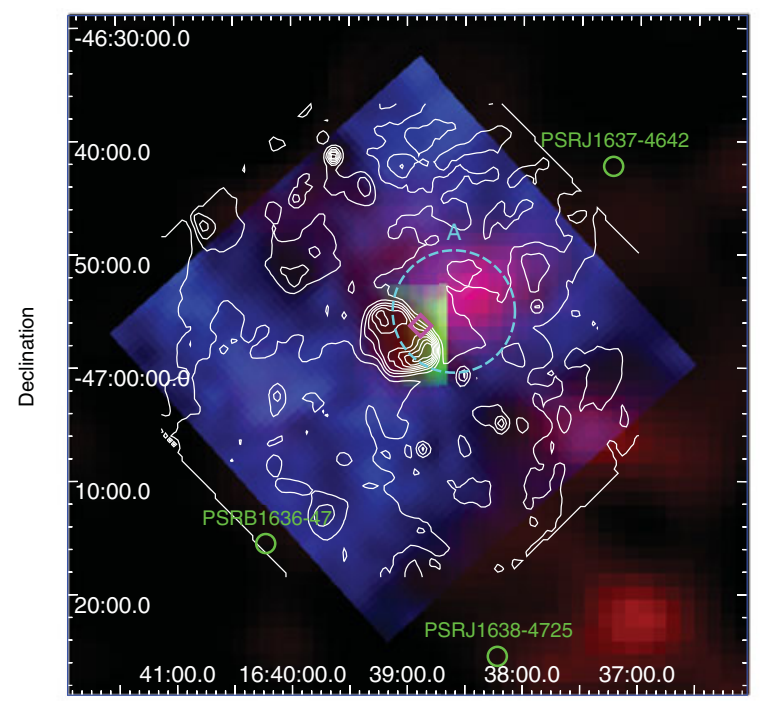

Right ascension (J2000)

Figure 2. Tricolor image of Kes 41. Red: Fermi-LAT 2-300GeV residual counts map with the emissions from the Galactic and extragalactic diffuse background as well as the 2FGL sources (except 2FGL J1638.0-4703c) deducted. Green: ${ }^{12} \mathrm{CO}(J=1-0)$ integrated emission $\left(V_{\mathrm{LSR}}=-70\right.$ to $\left.-40 \mathrm{~km} \mathrm{~s}^{-1}\right)$ with the field of view $11^{\prime} \times 10^{\prime}$. Blue: HI line emission from SGPS integrated map $\left(V_{\mathrm{LSR}}=-55\right.$ to $\left.-50 \mathrm{~km} \mathrm{~s}^{-1}\right)$. The green circles label the positions of known pulsars. The dashed circle, the diamond and the contours are the same as in Fig.1.

dense molecular gas (case B). We assumed the target gas with average number density $n_{\mathrm{t}}$, and the protons with a broken power-law distribution,

$$
d N_{\mathrm{p}} / d E_{\mathrm{p}} \propto E_{\mathrm{p}}^{-\alpha_{\mathrm{p}}}\left(1+\left(E_{\mathrm{p}} / E_{\mathrm{b}}\right)^{2}\right)^{-\Delta \alpha_{\mathrm{p}} / 2} .
$$

\section{The total energy in protons with energy above $1 \mathrm{GeV}$ is}

$$
W_{\mathrm{p}}(>1 \mathrm{GeV}) \sim 0.7 \times 10^{50} E_{51}\left(n_{\mathrm{t}} / 100 \mathrm{~cm}^{-3}\right)^{-1} \mathbf{e r g},
$$

where $E_{51}=E_{\mathrm{SN}} / 10^{51} \mathrm{erg}$ is the dimensionless SN explosion energy.

Case $C$. As shown in Fig.1, the centroid of the gamma-ray emission is outside the northwestern boundary of Kes 41 . Thus, the interaction of high-energy protons escaped from Kes 41 with the nearby MC (case C), is a very reasonable scenario for the origin of the gamma-rays. In this case, the energy distribution of protons in the finite volume of the adjacent cloud is obtained following the work of Li \& Chen (2012).

Case D. According to Zhang et al. (2015), Kes 41 is enclosed in a cavity that may send a reflected shock backward when the blast wave collides with it. It is necessary to consider the case that the reflected shock could accelerate particles effectively after the forward shock becomes radiative. We simplify this situation as a continuous proton injection from the SNR center and follow the prescription of Aharonian \& Atoyan (1996). In addition, the MC is regard as a point and the energy conversion fraction $\eta=0.1$ is adopted.

Comparison among different models. The parameters obtained for the above four cases via fitting the spectra of Kes 41 are listed in Table 1 (for the definition of the parameters and calculation details, see Liu et al. (2015), Li \& Chen (2012) and the references therein). For the leptonic scenario (case A), the total energy required in electrons with energy above $1 \mathrm{GeV}, W_{\mathrm{e}}(>1 \mathrm{GeV}) \sim 1.3 \times 10^{51} \mathrm{erg}$, is unreasonably high, i.e. of the order of the canonical SN explosion energy. As for the hadronic scenarios, the variation ranges of these parameters are reasonable given Kes 41's own conditions and its ambient environment. 


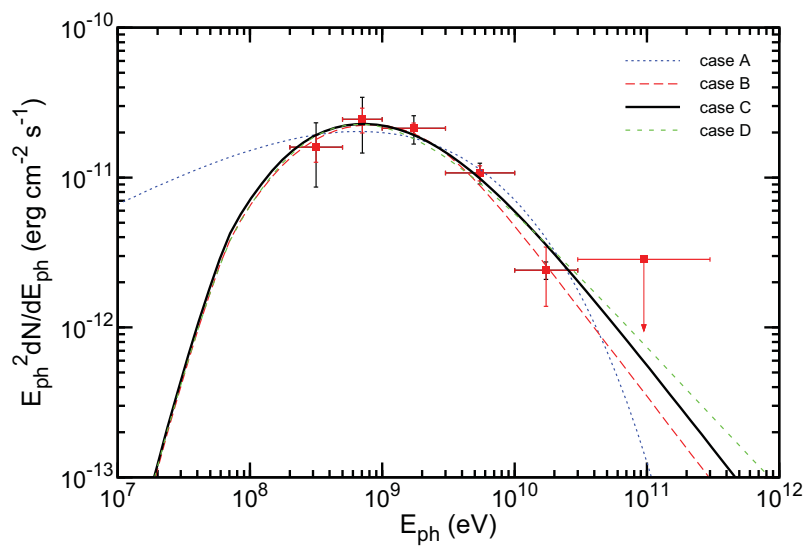

Figure 3. Fermi $\gamma$-ray spectral energy distribution of source A, fitted with various models (see text). The statistical errors are indicated by red bars and the systematic errors are indicated by black bars. For case C, $\mathrm{t}_{\text {age }}=10 \mathrm{kyr}$ and $\mathrm{R}_{c}=20 \mathrm{pc}$ and for case $\mathrm{D}, \mathrm{t}_{\text {age }}=10 \mathrm{kyr}$ (see Table $1)$.

Table 1. Model parameters for the emissions of SNR accelerated particles

\begin{tabular}{|c|c|c|c|c|c|c|}
\hline & & $\alpha_{\mathrm{e}}$ & & $\begin{array}{l}E_{\mathrm{e}, \mathrm{cut}} \\
(\mathrm{GeV})\end{array}$ & $\begin{array}{c}W_{\mathrm{e}}(>1 \mathrm{GeV}) \\
\left(10^{51} \mathrm{erg}\right)\end{array}$ & \\
\hline \multirow[t]{2}{*}{ case A } & & 2.0 & & 400 & 1.3 & \\
\hline & & $\alpha_{\mathrm{p}}$ & $\Delta \alpha_{\mathrm{p}}$ & $\begin{array}{c}E_{\mathrm{b}} \\
(\mathrm{GeV})\end{array}$ & $\begin{array}{c}n_{\mathrm{t}} E_{51}^{-1} W_{\mathrm{p}}(>1 \mathrm{GeV}) \\
\quad\left(10^{51} \mathrm{erg} \mathrm{cm}^{-3}\right)\end{array}$ & \\
\hline \multirow[t]{2}{*}{ case B } & & 2.0 & 1.2 & 18 & 7 & \\
\hline & $\begin{array}{l}t_{\text {age }} \\
(\mathrm{kyr})\end{array}$ & $\alpha_{\mathrm{p}}$ & $\delta$ & $\chi$ & $\begin{array}{l}\Delta R_{c} \\
(\mathrm{pc})\end{array}$ & $\begin{array}{c}M_{\mathrm{cl}} \\
\left(10^{4} M_{\odot}\right)\end{array}$ \\
\hline \multirow[t]{2}{*}{ case $\mathrm{C}$} & $\begin{array}{c}4 \\
10 \\
100\end{array}$ & $\begin{array}{l}2.4 \\
2.4 \\
2.4\end{array}$ & $\begin{array}{l}0.7 \\
0.7 \\
0.7\end{array}$ & $\begin{array}{c}0.07 \\
0.03 \\
0.004\end{array}$ & $\begin{array}{c}5 \\
10 \\
13\end{array}$ & $\begin{array}{l}4.5 \\
11 \\
18\end{array}$ \\
\hline & $\begin{array}{l}t_{\text {age }} \\
(\mathrm{kyr})\end{array}$ & $\alpha_{\mathrm{p}}$ & $\delta$ & $\chi$ & $\begin{array}{l}R_{c} \\
(\mathrm{pc})\end{array}$ & $\begin{array}{c}M_{\mathrm{cl}} \\
\left(10^{4} M_{\odot}\right)\end{array}$ \\
\hline case D & $\begin{array}{c}10 \\
10 \\
100\end{array}$ & $\begin{array}{l}2.4 \\
2.4 \\
2.4\end{array}$ & $\begin{array}{l}0.7 \\
0.7 \\
0.7\end{array}$ & $\begin{array}{l}0.25 \\
0.45 \\
0.05\end{array}$ & $\begin{array}{l}15 \\
20 \\
20\end{array}$ & $\begin{array}{l}18 \\
40 \\
40\end{array}$ \\
\hline
\end{tabular}

Taking the case $\mathrm{B}$ as an example, if the mean target density $n_{\mathrm{t}} \sim 100 \mathrm{~cm}^{-3}$, then the total energy in protons with energy above $1 \mathbf{G e V}, W_{\mathrm{p}}(>1 \mathrm{GeV})$ is $\sim 1 \times$ $10^{50} E_{51} \mathrm{erg}$, i.e. the fraction, $\eta$, of the SN explosion energy converted into protons is of the order of 0.1. As shown in Fig. 3, the fitting effect of case A is less satisfactory, and the escape cases (case C/D) have harder model spectra above $10 \mathrm{GeV}$ than the interaction-at-the-shock case (case B). Further TeV observations will likely be helpful to distinguish the two scenarios. Although both the interaction at the shock and the illumination by escaping protons, can generally explain the gamma-ray properties of source A, the latter cases (C/D) are preferred as the $\mathrm{GeV}$ emission is offset from the SNR itself and centered on the SNR-MC interaction region. 


\section{Summary}

Using 5.6 years of Fermi-LAT observation data, we have detected a gamma-ray source to the northwest of SNR Kes 41 with a significance of $24 \sigma$ in $0.2-300 \mathrm{GeV}$. Neither significant long-term variability nor periodicity is detected from the timing analysis of source $\mathrm{A}$ in the same energy range. The best-fit location of the gamma-ray emission is within the region of the MC with which the SNR interacts. Although the spectrum is similar to those of pulsars, there is no known radio pulsar within the $3 \sigma$ location uncertainty that could be responsible for the high $\mathrm{GeV}$ luminosity. The energy budget would be too high if the gamma-ray emission was produced by high energy electron inverse Compton scattering off the CMB photons. The decay of neutral pions produced by the collision between relativistic protons accelerated by the shock of Kes 41 and the adjacent MC, can naturally explain the origin of the gamma-ray emission. Interaction of high-energy protons escaped from the SNR shock front with the adjacent MC seems more consistent with the Fermi-LAT observations presented in this article.

\section{References}

Abdo, A. A., Ackermann, M., Ajello, M., et al. 2009, ApJ (Letters), 706, L1

Abdo, A. A., Ackermann, M., Ajello, M., et al. 2010a, ApJ, 718, 348

Abdo, A. A., Ackermann, M., Ajello, M., et al. 2010b, Science, 327, 1103

Abdo, A. A., Ackermann, M., Ajello, M., et al. 2010c, ApJ, 712, 459

Abdo, A. A., Ackermann, M., Ajello, M., et al. 2013, ApJS, 208, 17

Ackermann, M., Ajello, M., Allafort, A., et al. 2013, Science, 339, 807

Aharonian, F. A. \& Atoyan, A. M. 1996, A\&A, 309, 917

Atwood, W. B., et al. 2009, ApJ, 697, 1071

Blandford, R. D. \& Cowie, L. L. 1982, ApJ, 260, 625

Castro, D., Slane, P., Carlton, A., \& Figueroa-Feliciano, E. 2013, ApJ, 774, 36

Caswell, J. L. 2004, MNRAS, 349, 99

Gabici, S., Aharonian, F. A., \& Casanova, S. 2009, MNRAS, 396, 1629

Ginzburg, V. L. \& Syrovatskii, S. I. 1969, The origin of cosmic rays (New York: Gordon and Breach)

Koralesky, B., Frail, D. A., Goss, W. M., Claussen, M. J., \& Green, A. J. 1998, AJ, 116, 1323

Li, H. \& Chen, Y. 2010, MNRAS, 409, L35

Li, H. \& Chen, Y. 2012, MNRAS, 421, 935

Nolan, P. L., Abdo, A. A., Ackermann, M., et al. 2012, ApJS, 199, 31

Ohira, Y., Murase, K., \& Yamazaki, R. 2011, MNRAS, 410, 1577

Tang, X. \& Chevalier, R. A. 2014, ApJ (Letters), 784, L35

Uchiyama, Y., Blandford, R. D., Funk, S., Tajima, H., \& Tanaka, T. 2010, ApJ (Letters), 723, L122

Wenger, M., Ochsenbein, F., Egret, D., et al. 2000, A\&AS, 143, 9

Whiteoak, J. B. Z. \& Green, A. J. 1996, A\&AS, 118, 329

Xing, Y., Wang, Z., Zhang, X., \& Chen, Y. 2014, ApJ, 781, 64

Zhang, G.-Y., Chen, Y., Su, Y., Zhou, X., Pannuti, T. G., \& Zhou, P. 2015, ApJ, 799, 103 